Rapid Reviews COVID-19

\title{
Review 2: "Inhaled budesonide in the treatment of early COVID- 19 illness: a randomised controlled trial"
}

Ariel Berlinski ${ }^{\mathbf{1}}$

${ }^{1}$ Professor, University of Arkansas for Medical Sciences, Pediatrics

Published on: Apr 26, 2021

DOI: $10.1162 / 2 \mathrm{e} 3983 f 5.182368 \mathrm{a} 8$

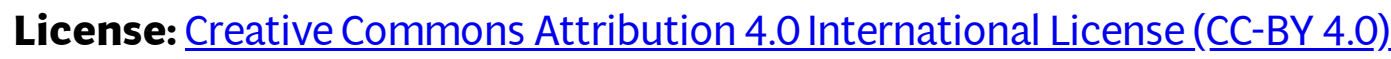




\section{$\underline{\text { RR:C19 Evidence Scale rating by reviewer: }}$}

- Potentially informative. The main claims made are not strongly justified by the methods and data, but may yield some insight. The results and conclusions of the study may resemble those from the hypothetical ideal study, but there is substantial room for doubt. Decision-makers should consider this evidence only with a thorough understanding of its weaknesses, alongside other evidence and theory. Decisionmakers should not consider this actionable, unless the weaknesses are clearly understood and there is other theory and evidence to further support it.

*****************************************

\section{Review:}

The authors hypothesized that the low number of asthma/COPD patients affected with COVID could be due to a protective effect exerted by the use of inhaled corticosteroids. They conducted a randomized, open-label, parallel study of inhaled Budesonide versus usual care. The study was stopped by the DSMB.

The authors should address the following issues:

\section{METHODS}

1. Please justify the choice of open-label vs. placebo-controlled design

2. Was the need for urgent care visits decided per protocol or left up to the subjects?

\section{RESULTS}

3. Please discuss why you did not see a difference in viral load. This goes against your rationale on how the drug interferes with viral replication. Moreover, Spagnuolo et al. retrospectively reviewed the effect of steroids in moderate to severe COVID and did not find differences in time to PCR negativization. Fang et al. also reported that the use of steroids did delay viral clearance in COVID 19 patients. Please speculate on the mechanism of action of budesonide in light of these findings.

\section{DISCUSSION}

4. Please clarify: your abstract states that the study was stopped by the drug safety monitoring board. However, the discussion states that it was stopped due to national pandemic control measures, and national prioritization rules in the UK. 
5. The authors need to state that their findings need to be confirmed in a randomized, double-blind placebo-controlled trial. 